\title{
Imaging and spectroscopy of the LMC He II nebula N 44C and its ionizing star
}

\author{
Vanessa C. Galarza \\ New Mexico State University, Las Cruces, NM 88003, USA \\ Donald R. Garnett \\ University of Arizona, Tucson, AZ 85721, USA \\ You-Hua Chu \\ University of Ilinois, Urbana, IL 61801, USA
}

\begin{abstract}
We present results from new HST imaging and spectroscopy of the peculiar Large Magellanic Cloud H II region N 44C and its ionizing star. While this nebula exhibits strong $\mathrm{He}$ II recombination emission, the source of the $\mathrm{He}^{+}$ ionizing photons has not been found. The UV spectrum of the ionizing star suggests an approximate spectral class of O7-O8; the UV Si IV , He II , and N IV features do not show P-Cygni profiles, indicating that the ionizing star is not a supergiant. No companion star has yet been detected. Ground-based and HST optical spectroscopy of the ionized gas shows that the nebular abundances of $\mathrm{C}$, $\mathrm{N}, \mathrm{O}$ and $\mathrm{He}$ are not anomalous relative to other LMC H II regions, suggesting that no previous WR/SN companion has disappeared. Echelle spectroscopy has also ruled out the presence of high velocity shocked gas. Deep ROSAT imaging shows no X-ray point source in this location. The "fossil X-ray binary" hypothesis of Pakull \& Motch (1989) remains the best explanation for the ionization of this nebula; however, convincing evidence for this hypothesis remains elusive.
\end{abstract}

\section{Introduction}

A handful of $\mathrm{H}$ II regions with strong He II recombination emission have been discovered over the past decade (Garnett et al. 1991). The He II emission is peculiar because of the high photon energies $(h \nu \geq 54 \mathrm{eV})$ required to further ionize $\mathrm{He}^{+}$. NLTE stellar atmosphere models predict that a star capable of ionizing helium to this state must have $T_{\text {eff }} \gtrsim 60000 \mathrm{~K}$. Most of these $\mathrm{H}$ II regions discovered to have their own "He II regions" appear to be associated with WolfRayet stars or massive X-ray binary systems. However, Stasińska et al. (1986) found that the central engine ionizing $\mathrm{N} 44 \mathrm{C}$ in the LMC appears to be a normal O star.

A number of theories have been proposed to solve the problem of $\mathrm{N} 44 \mathrm{C}$. The He II observed in the optical spectrum of the nebula may be accounted for by a $T_{\text {eff }} \simeq 50000 \mathrm{~K}$ O-type supergiant near the Eddington-limit. Radiative shocks have also been proposed as the source of the high ionization. Pakull \& Motch (1989) have suggested that the high degree of ionization found in this 
nebula could be caused by a fossil X-ray binary which has turned off within the last century.

\section{Observational data}

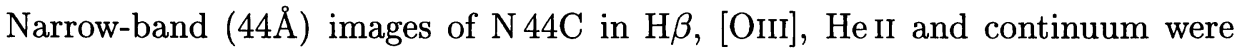
obtained with the CTIO $0.9 \mathrm{~m}$ in 1991 . We also obtained $H S T$-wFPC2 images in F502N, F656N and F547N. Optical long-slit spectra of the N 44C nebula were taken using the RC spectrograph on the CTIO $4.0 \mathrm{~m}$ telescope and with the HST-FOS. A UV spectrum of "Star 2", the only apparent ionizing source in $\mathrm{N} 44 \mathrm{C}$, was obtained with HST-GHRS. An echellogram and ROSAT X-ray image were provided by Magnier et al. (1996).

\section{Results}

- $\mathrm{N} 44 \mathrm{C}$ is an LMC H II region with its own 3 pc diameter He II region; the source of the $\mathrm{He}^{+2}$ ionizing photons is unknown.

- The central star within $\mathrm{N} 44 \mathrm{C}$ is classified as an O7-O8 main sequence star based on the absence of Si IV and CIV P-Cygni features in its UV spectrum.

- Analysis of CTIO and HST-FOS spectra reveal a chemical composition similar to that typically found in the LMC; this rules out the hypothesis that a previous WR/SN has produced the high level of ionization.

- Echelle spectroscopy rules out the existence of high velocity gas, excluding shock ionization as the source for the He II emission.

- ROSAT X-ray imaging shows no point source in this region; no X-ray binary system is detected to low limits. This does not exclude the possibility that a "fossil" X-ray binary system is responsible for the high ionization level.

- Analysis of the ionic ratios found in the gas and further analysis of the stellar spectrum, will aid in the modelling of the central ionizing source.

\section{References}

Garnett, D.R., Kennicutt, R.C. Jr., Chu, Y.-H., Skillman, E.D. 1991, ApJ 373, 458 Magnier, E.A., Chu, Y.-H., Points, S.D., Hwang, U., Smith, R.C. 1996, ApJ 464, 829

Pakull, M.W., Motch, C. 1989, Nature 337, 337

Stasińska, G., Testor, J.G., Heydari-Malayeri, M. 1986, A\&A 170, L4 